\title{
DYNAMIC LOCAL TRACING FOR 3D AXON CURVILINEAR STRUCTURE DETECTION FROM MICROSCOPIC IMAGE STACK
}

\author{
Jun Wang ${ }^{\mathrm{a}, \mathrm{b}}$, Xiaobo Zhou ${ }^{\mathrm{a}, \mathrm{c}}$, Ju Lu ${ }^{\mathrm{d}}$, Jeff Lichtman ${ }^{\mathrm{d}}$, Shih-Fu Chang ${ }^{\mathrm{b}}$, Stephen T.C. Wong ${ }^{\mathrm{a}, \mathrm{c}}$

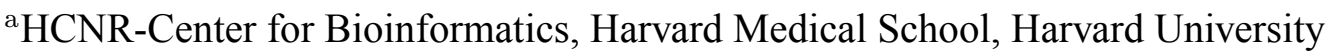 \\ ${ }^{\mathrm{b}}$ Department of Electrical Engineering, Columbia University \\ ${ }^{\mathrm{c}}$ Functional and Molecular Imaging Center, Department of Radiology, Brigham and Woman's Hospital \\ ${ }^{\mathrm{d}}$ Department of Molecular and Cellular Biology, Harvard University
}

\begin{abstract}
To study the morphologic structure of axons can help neuroscientists understand the neuronal function and development. The modern microscopes provide the fundamental tool for visual inspection of axonal structure. Due to the high volume of generated microscopic axon image data, it is critical to develop an automated technique for robustly and rapidly detecting 3D axonal structure. In this paper, we present a pure 3D approach to extract the curvilinear structure of axonal axes from microscopic image stacks. The method mimics the axon tracing procedure in $3 \mathrm{D}$ space as walking along a path with minimized cost value, which corresponds to the shortest path problem (SPP) in graph theory. The global solution for SPP, such as Dijkstra's algorithm, is infeasible for the real axon tracing problem because of the computation cost. We simplify this problem using a dynamic local tracing technique with linear computation complexity. The merits of the proposed method lie in that it can handle the short turn and non-vertical problems and also can separate closely distributed axons from each other.
\end{abstract}

\section{INTRODUCTION}

Revealing the underlying geometric structure of axons, such as the branching and topology, can help the neuroscientist obtain a better understanding of the functional and developmental biology and neurodegeneration conditions [1]. The modern advancements in high-resolution and fast microscopic technique provide scientists the visual perception of axons in threedimensional space through a series of image slices, which is so called microscopic image stack. Due to the high content confocal microscopy images, the manual processing brings extremely heavy work load and is infeasible for wide-scale axons morphological analysis. Therefore, it is crucial and emergent to develop a rapid and robust technique to segment the axonal structures from large volume of microscopic image stacks. There have been some approaches proposed to extract the geometric structure of tubular biological objects, such as blood vessels and human airway tree in clinic research [2] [3]
[4]. Moreover, some recent literatures reported the progress on extracting the axons 3D structure, such as the repulsive force based snake model [5][6][7]. However, most of the proposed methods for 3D morphological structure extraction utilize 2D image based object segmentation and the association between successive image slices.

There are two categories of procedures for these kinds of $2 \mathrm{D}$ based techniques. One is first to segment the object of interest in each image slice and then find the object associations. The other is to extract the objects in the first image slice and then conduct tracking procedure on the consequent slices. The main constraint lies in that it assumes the 3D curvilinear structures are approximately perpendicular to the imaging plane. Particularly, these 2D based methods could completely fail when segmenting the axons with short turn and parallel parts to the scanning plane. Furthermore, due to the connection complexity and low-contrast signal of axons, the axon segmentation and object correspondence encounter difficulties for many slices in the microscopic image stacks.

In this paper, we present a $3 \mathrm{D}$ space based approach for extracting the geometric structure from confocal microscopy images of axons. The basic idea is to mimic a walking procedure in the $3 \mathrm{D}$ space through a tubular routeway with minimal cost. The finding of the path with minimal cost can be formulated by the classical shortest path problem (SPP) in graph theory. However, the state-of-art solutions for SPP, such as Dijkstra's algorithm, have the computation complexity no less than $\mathcal{O}(E+V \log V)$, where $E$ is the number of edges and $V$ is the number of vertex in the graph [8]. It cannot satisfy the real problem of axon segmentation because of the huge volume of data and complex connection of axons. Motivated by the well-known Gestalt psychology laws of closure in cognitive science, proximity and continuity for $2 \mathrm{D}$ salient objects [9], we formulate our cost function to reflect the saliency of 3D axonal axes based on the local characteristics of axons in three aspects: smoothness, proximity and continuity. Finally we try to solve the optimization problem by local dynamic searching with linear time computational cost instead of the expensive global optimization. The exper- 


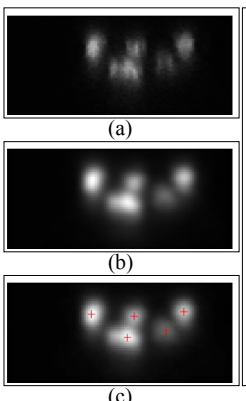

(c)

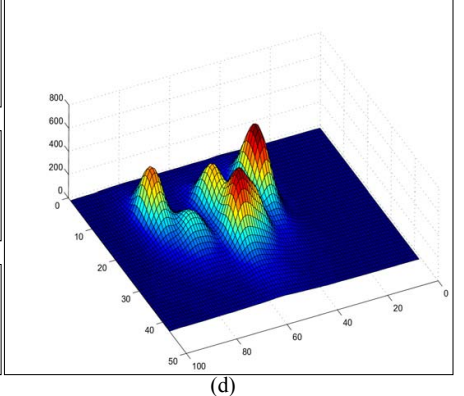

Fig. 1. The preprocessing and tracing initialization of axon slice: (a) original axon image patch; (b) after thresholding and Gaussian smoothing; (d) initialized axon centroid; and (d) corresponding image terrain surface of (b). The centroids are corresponding to the peaks in the surface.

imental results of curvilinear structure extraction of axonal axes from real microscopy image stack empirically validate our proposed method.

\section{METHODOLOGY}

\subsection{Preprocessing and tracing initialization}

Due to the existing background noise, the first step of preprocessing is to use thresholding technique to remove the noise (an axon patch of raw image shown in Fig.1.a). This denoising processing can provide a cleaner axon presentation and also reduce the computation cost in the following dynamic tracing.

For the tracing initialization, the most important geometric information is the centroid location of each axon in the first slice. In joint space-range domain, the discrete image can be treated as terrain surface, whose height is the intensity of the pixel. After the thresholding and Gaussian smoothing processing (Fig.1.b), the axons is shown in the form of terrain surface (Fig.1.d), where each axon is corresponding to one "Hill". Obviously, the peak position in the terrain surface is the exact centroid position of each axon. The mean shift technique can be applied to locate those centroids [10]. The results are displayed in Fig.1.c.

\subsection{Definition of tracing cost}

Here we define the cost function to describe the local neuronal topology considering three factors: smoothness, proximity and continuity. Smoothness property relates to the shape requirement of the extracted central line. Proximity means the gap between each pair of transition pixels should be small. Continuity means the energy or intensity along the central line should be smoothly distributed. Balancing the three cost factors, the central line can be extracted along the optimized path. These three local axonal properties are defined as follows.

Smoothness: Assume the image stack is described by $I(x, y, z)$, we can calculate the three-dimensional Hessian matrix as:

$$
H(I)=\left[\begin{array}{lll}
I_{x x} & I_{x y} & I_{x z} \\
I_{y x} & I_{y y} & I_{y z} \\
I_{x z} & I_{y z} & I_{z z}
\end{array}\right]
$$

where $I_{x x}, I_{y y}, I_{z z}, I_{x y}, I_{y z}, I_{x z}$ are the second-order partial differentials of $I(x, y, z)$. The estimation of discrete case partial differentials can be obtained by conducting convolution with the partial derivatives of Gaussian kernel. For instance, the second partial differential along $x$ axis $I_{x x}$ can be approximated as:

$$
I_{x x}=\frac{\partial^{2} G(x, y, z ; \Sigma)}{\partial x^{2}} * I(x, y, z)
$$

where $G(x, y, z)$ is a $3 \mathrm{D}$ Gaussian function with covariance matrix $\Sigma$ and zero mean. The eigenvalue decomposition of $H(I)$ can derive the largest eigenvalue $\lambda_{\max }$ and the corresponding eigenvector $\mathbf{e}_{\max }$, which denotes the direction with the maximum gradient of intensity. Simply, we use $\mathbf{e}_{\max }$ in three dimensional space as the signature of direction of the axons in a certain position. Hence we define the smoothness between two points $\mathbf{v}_{i}=\left\{x_{i}, y_{i}, z_{i}\right\}$ and $\mathbf{v}_{j}=\left\{x_{j}, y_{j}, z_{j}\right\}$ as:

$$
s_{\mathbf{v}_{i}, \mathbf{v}_{j}}=\frac{\left\|\mathbf{e}_{\max }^{i}\right\| \cdot\left\|\mathbf{e}_{\max }^{j}\right\|}{<\mathbf{e}_{\max }^{i} \cdot \mathbf{e}_{\max }^{j}>}
$$

Proximity: The definition of proximity is intuitive, which reflects the geometric distance between two adjacent pixels along the axonal axis. Here we just use the Euclidian distance to represents the proximity, which is calculated as:

$$
p_{\mathbf{v}_{i}, \mathbf{v}_{j}}=\left\|\mathbf{v}_{i}-\mathbf{v}_{j}\right\|
$$

Continuity: Assuming that the intensity distributed along the axonal axis should smoothly change in the $3 \mathrm{D}$ space, we can evaluate the local continuity property directly based on the intensity of pixels. However this criterion will be easily affected by noise. We utilize the density of intensity instead of intensity of pixels. Regard the axons as the 3D objects with mass density in different locations. Each pixel $\mathbf{v}=(x, y, z)$ in the axonal image stack is treated as a unit $3 \mathrm{D}$ cubic with the mass $I(\mathbf{v})$. We apply the multivariate kernel density to estimate the density at position $\mathbf{v}_{0}$ with kernel $K(\mathbf{v})$ and homogeneous kernel size $h$ [10]:

$$
f\left(\mathbf{v}_{0}\right)=\frac{1}{n h^{3}} \sum I(\mathbf{v}) \cdot K\left(\frac{\mathbf{v}_{0}-\mathbf{v}}{h}\right)
$$

$n$ is the number of pixels in the sphere region $\left\|\mathbf{v}-\mathbf{v}_{0}\right\| \leq h$. The optimal 3D kernel with minimum mean integrated square error is Epanechnikov kernel [11]:

$$
K_{E}(\mathbf{v})= \begin{cases}\frac{5}{2} a^{-1}\left(1-\mathbf{v}^{T} \mathbf{v}\right) & \text { if } \mathbf{v}^{T} \mathbf{v}<1 \\ 0 & \text { otherwise }\end{cases}
$$


where $a=\frac{4}{3} \pi h^{3}$ represents the volume the sphere. So the continuity between pixels $\mathbf{v}_{i}, \mathbf{v}_{j}$ is: $c_{\mathbf{v}_{i}, \mathbf{v}_{j}}=\left|f\left(\mathbf{v}_{i}\right)-f\left(\mathbf{v}_{j}\right)\right|$.

Finally, given two adjacent non-zero pixels $\mathbf{v}_{i}, \mathbf{v}_{j}$, the total local tracing cost is defined as:

$$
g_{\mathbf{v}_{i}, \mathbf{v}_{j}}=s_{\mathbf{v}_{i}, \mathbf{v}_{j}}+\eta \cdot p_{\mathbf{v}_{i}, \mathbf{v}_{j}}+\mu \cdot c_{\mathbf{v}_{i}, \mathbf{v}_{j}}
$$

where the coefficients $\eta, \mu$ are used to balance the weight of the three different costs.

\subsection{Dynamic local tracing}

The objective of extracting the axonal axis is to find a path $\mathcal{P}$ consisting of a sequence of successive pixels $\left\{\mathbf{v}_{i}\right\}, i=$ $1,2, \ldots, N$, in the image stack with the minimum cost:

$$
\mathcal{P}_{\text {opt }}=\min _{\mathcal{P}} \sum_{i=1}^{N-1}\left(s_{\mathbf{v}_{i}, \mathbf{v}_{i+1}}+\eta \cdot p_{\mathbf{v}_{i+1}, \mathbf{v}_{i+1}}+\mu \cdot c_{\mathbf{v}_{i}, \mathbf{v}_{i+1}}\right)
$$

The solution of Eq. 8 can be treated as a classical shortest path problem (SPP), given start vertex and unfixed end vertex. However this solution is not feasible for the axon problem due to the high volume of data. A typical axonal image stack with the scale of $482 \times 43 \times 512$ contains more than $10^{5}$ nonzero pixels after thresholding processing. Alternatively, we transfer the global path searching procedure to a dynamic and local optimization problem as:

$$
\mathcal{P}_{\text {opt }}=\left\{\mathbf{v}_{i}\right\}=\sum_{i=1}^{N-1} \min _{\mathbf{v}_{i+1}}\left(s_{\mathbf{v}_{i}, \mathbf{v}_{i+1}}+\eta \cdot p_{\mathbf{v}_{i}, \mathbf{v}_{i+1}}+\mu \cdot c_{\mathbf{v}_{i}, \mathbf{v}_{i+1}}\right)
$$

From Eq. 9, the optimal tracing path is determined by local optimization searching. The local search procedure is iteratively repeated from the start point to find the adjacent optimal pixel until reach the boundary of the image stack. For each local search operation, the pixel in the hemisphere region with radius $r$ along the axonal direction will be exhaustedly evaluated to find the best matching.

Instead serially tracing the axons one by one in the axonal image stack, we execute a parallel tracing strategy. Therefore, the total cost for all the axons need to be minimized for each local tracing step. Moreover, the parallel tracing provides the merit for detecting tracing contradiction when more than one axon merges to the same successive vertex. In that case, the reverse tracing procedure will be applied to find the most reliable previous axonal vertex of the merging point.

\section{CASE STUDY}

Data: The confocal microscopic images stack used in our experiment has the dimensionality with $482 \times 43 \times 512$, which contains 5 axons. Fig. 2 shows several slices of the axons image stacks. From the figure, we observed the following characteristics of the real axon data. First the appearances of the

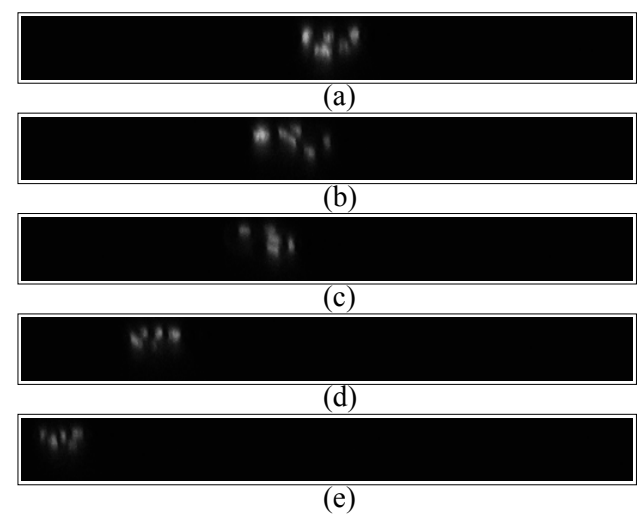

Fig. 2. The axonal image slices samples. From top to bottom, the slices are indexed as 1, 71,121,321, and 471 in the microscopic image stack.

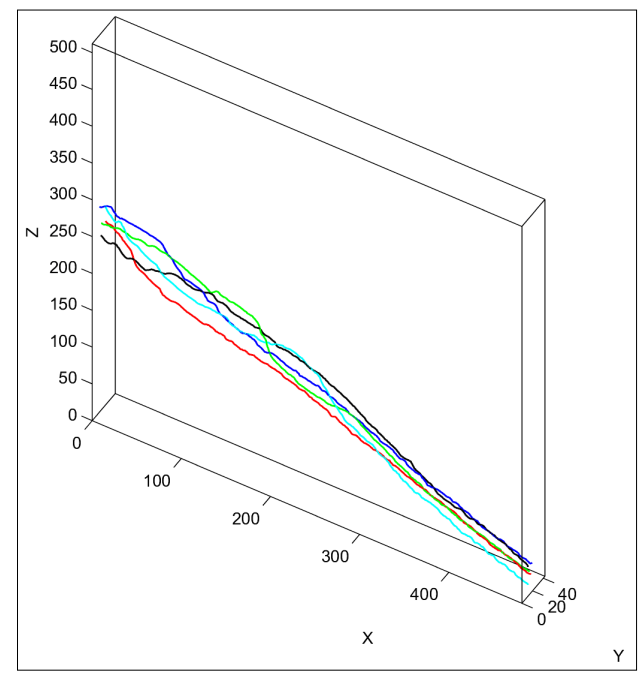

Fig. 3. The detected 3D curvilinear structure of axonal axes of real axon image stack.

axons are severely diverse for different slices. Second, the axons are very close or even touch together in some slices. Third, in some slice, the axon could be invisible or mixed together (Slice 121 and 321 shown in Fig.2).

Results: As described in Section 2.1, the initial position of axonal center is automatically located by mean shift approach. Given these start points, the five axons are traced in the same time. The reverse tracing strategy is applied when encountering the axon merging problem because here we assume that the image stack dose not contain branching and merging axons. Finally we get a series of tracing points for each axon. Figure 3 gives the $3 \mathrm{D}$ visualization results of the traced curvilinear structure of axonal axes. The touching together axons are well separated by the proposed method.

Validation: The extracted curvilinear structure of axonal axes was validated by comparing with the manual labeling re- 


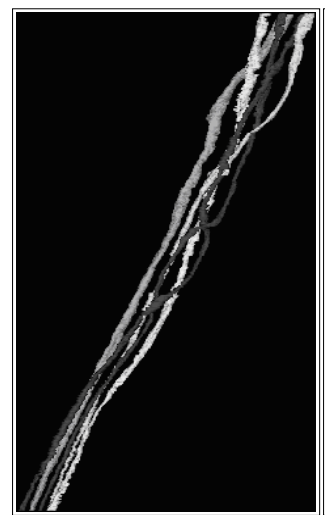

(a)

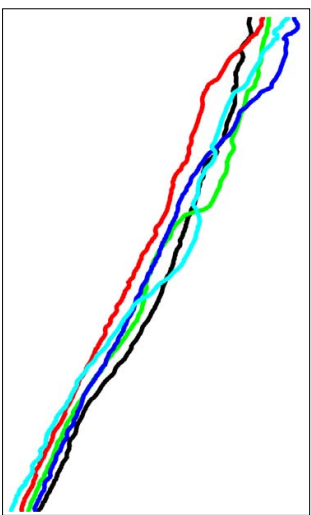

(b)
Fig. 4. Validation by comparing with manual results on $X-Z$ projection: (a) is the manual result and (b) is the automatic result.

sult. The neurological expert provides the manual results on the $\mathrm{X}-\mathrm{Z}$ plane, as shown in Fig.4a. Meanwhile, we project our extracted $3 \mathrm{D}$ axonal axes onto $\mathrm{X}-\mathrm{Z}$ plane and obtain the result as Fig.4b. The visual comparison shows that our result is very close to the manual effort, although we have not presented quantitative evaluation comparing with the manual ground truth in the current work.

\section{CONCLUSION AND FUTURE WORK}

In this paper, we present a pure 3D space approach for extracting the curvilinear structure of multiple axonal axes from microscopic image stacks. The microscopic image stack is treated as the $3 \mathrm{D}$ distributed pixels. The segment of the axonal axes is formulated as finding a path with optimal cost. The cost function between two adjacent pixels in the axonal data is defined based on three basic rules, i.e., smoothness, continuity and proximity. Although this SPP has the global solution in graph theory, we have to solve this problem using local tracing method due to the extremely expensive computation cost. Our proposed local dynamic tracing approach has two distinguished innovations. First, we conduct parallel tracing for all the existing axons instead of serial tracing one by one. Second, we apply reverse tracing to solve the merging problem, which is resulted from touching together axons often seen in the real data. Moreover, our approach is completely based on the $3 \mathrm{D}$ geometric space. This merit can fundamentally solve the extraction of the axonal structure with non-vertical or even parallel scanned segments. Usually, most of the 2D methods could fail when tracing parallel scanned or short turned tubular objects. Based on the detected axonal axes, our future work is to apply model based method to reconstruct the axonal shape. It provides more geometric metrics, such as length, radius, and surface normal, which gives more quantitative information for axon function analysis. Moreover, tracing more complex axon structures (merg- ing, branching, etc.) is another research topic of importance.

\section{Acknowledgement}

This work was supported by a program grant to S. T. C. Wong from Harvard Center for Neurodegeneration and Repair.

\section{REFERENCES}

[1] N. Kasthuri and J.W. Lichtman, "The role of neuronal identity in synaptic competition," Nature, vol. 424, pp. 426-430, 2003.

[2] L. Antiga, B. Ene-Iordache, and B. Remuzzi, "Computational geometry for patient-specific reconstruction and meshing of blood vessels from angiography," IEEE Trans. on Med. Imaging, vol. 22, pp. 1529-1539, 2003.

[3] J. Tschirren, E. A. Hoffman, G. McLennan, and M. Sonka, "Intrathoracic airway trees: segmentation and airway morphology analysis from low-dose ct scans," IEEE Trans. on Med. Imaging, vol. 24, pp. 1529-1539, 2005.

[4] D. Aykac, E. A. Hoffman, G. McLennan, and J. M. Reinhardt, "Segmentation and analysis of the human airway tree from $3 \mathrm{~d} x$-ray ct images," IEEE Trans. on Med. Imaging, vol. 22, pp. 940-950, 2005.

[5] S. Schmitt, J. F. Evers, C. Duch, M. Scholz, and K. Obermayer, "New methods for computer-assisted 3d reconstruction of neurons from confocal image stacks," Neuroimage, vol. 23, pp. 1283-1298, 2004.

[6] H. Cai, X. Xu, J. Lu, J. Lichtman, S. P. Yung, and S. T. C. Wong, "Shape-constrained repulsive snake method to segment and track neurons in 3d microscopy images," in Proc International Symposium of Biomedical Imaging, Arlington, VA, USA, 2006.

[7] H. Cai, X. Xu, J. Lu, J. Lichtman, S. P. Yung, and S. T. C. Wong, "Repulsive force based snake model to segment and track neurons in $3 \mathrm{~d}$ microscopy image stacks," Neuroimage, vol. 32, pp. 1680-1620, 2006.

[8] T. H. Cormen, C. E. Leiserson, R. L. Rivest, and C. Stein, Introduction to algorithms, Second Edition, MIT Press and McGraw-Hill, 2001.

[9] K. Koffka, Principles of gestalt psychology, Harcourt, Brace and Company, 1935.

[10] D. Comaniciu and P. Meer, "Mean shift: A robust approach toward feature space analysis," IEEE Trans. on PAMI, vol. 24, pp. 603-619, 2002.

[11] B.W. Silverman, Density estimation for statistic and data analysis, Chapman \& Hall, 1986. 